\title{
Pancreatic metastasis of papillary thyroid carcinoma with an intraductal growth pattern
}
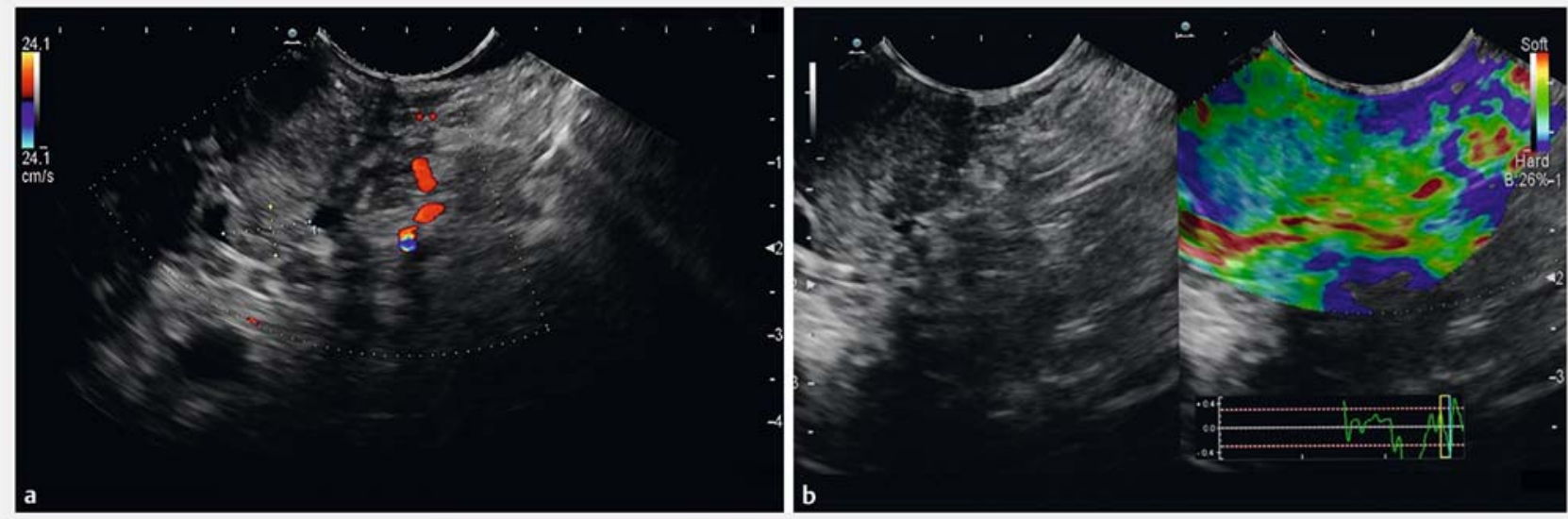

- Fig. 1 Endoscopic ultrasound (EUS) images. a Main pancreatic duct lesion invasion. b EUS elastography showed a soft pattern, different from pancreatic adenocarcinoma or neuroendocrine pancreatic tumors, which have a rigid elastography pattern.

Papillary thyroid carcinoma (PTC) is generally associated with excellent longterm outcome and survival [1,2]. PTC recurrence is most common in regional cervical lymph nodes and only $5 \%-7 \%$ of patients show a distant disease, most commonly in lung, bone, and brain [3].

Pancreatic PTC metastasis represents an extremely rare event and endoscopic ultrasound (EUS) plays a fundamental role in diagnosis, histological characterization, and therapeutic decision making $[4,5]$.

A 60-year-old man presented with a pancreatic solid mass (evidenced by computed tomography and magnetic resonance imaging) after recurrent pancreatitis. He had undergone thyroidectomy 15 years earlier for PTC and two subsequent extended lymphadenectomies for lymph node metastases. Subsequently, he presented paratracheal, pulmonary, and brain metastases.

EUS was performed using a linear echoendoscope (EG3870UTK; Pentax Medical, Tokyo, Japan) with the patient in the left lateral position and under deep sedation. A solid hypoechoic and hypervascular 20-mm lesion (\$Fig.1) was apparent in the pancreatic head, with

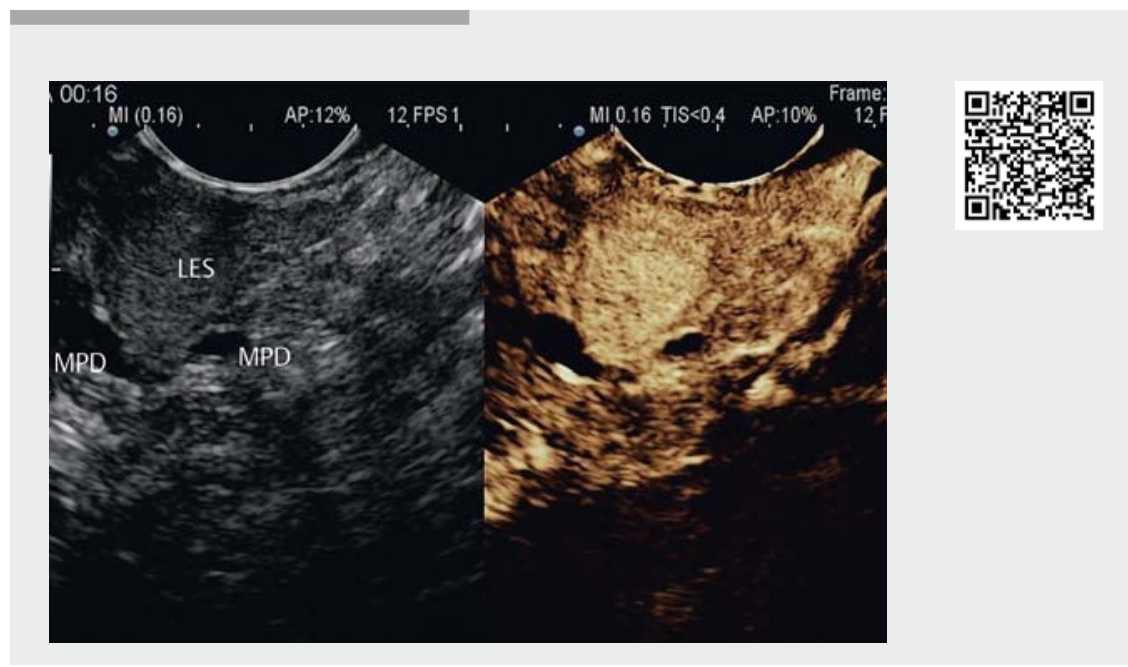

$\checkmark$ Video 1 Main pancreatic duct (MPD) invasion with hyperenhancement of pancreatic metastasis (LES), both in early and late phases. Intraductal lesion invasion is also well evidenced after intravenous contrast injection.

peculiar intraductal growth in the dorsal duct and main pancreatic duct (MPD), and initial dilation upstream ( $\vee$ Video 1 ). Fine-needle aspiration (25 G Expect SlimLine; Boston Scientific, Marlborough, Massachusetts, USA) and biopsy (25 G Acquire; Boston Scientific) were performed with rapid on-site evaluation by an expert cytopathologist. The final pathological diagnosis was PTC pancreatic metastasis.

At 2 months after the first EUS, the patient was evaluated for possible radiofrequency ablation (RFA) of the lesion under EUS guidance; however, pancreatic metastasis had increased in size and 


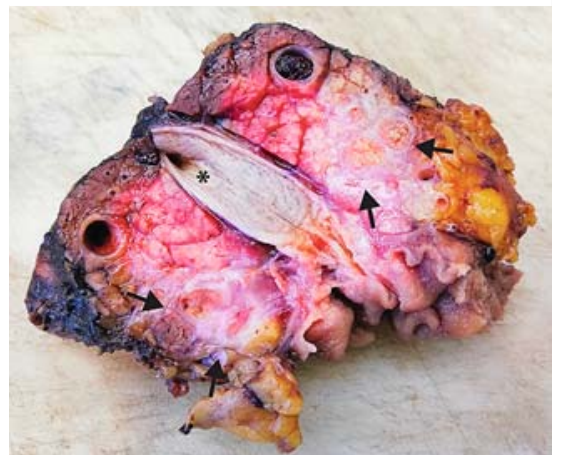

Fig. 2 Pancreaticoduodenectomy specimen after bivalving through the common bile duct (asterisk). Black arrows indicate the papillary tumor growing inside the main pancreatic duct.

complete MPD infiltration (associated with dorsal pancreatic duct infiltration) was apparent. Owing to the high risks of EUS-RFA (pancreatitis, duct stenosis, incomplete targeting of the lesion), the patient was finally referred for surgical

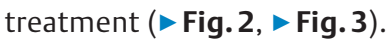

EUS represents a fundamental procedure for the diagnosis, characterization, and eventual treatment of pancreatic PTC metastasis. Surgery in high-volume centers can be considered in symptomatic patients.

Endoscopy_UCTN_Code_CCL_1AF_2AZ_3AB

\section{Competing interests}

The authors declare that they have no conflict of interest.

The authors

Gemma Rossi ${ }^{1}$, Maria Chiara Petrone ${ }^{1}$, Marco Schiavo Lena ${ }^{2}$, Claudio Doglioni ${ }^{2}$, Nicolò Pecorelli $^{3}$, Massimo Falconi ${ }^{3}$, Paolo Giorgio Arcidiacono ${ }^{1}$

1 Pancreato-Biliary Endoscopy and Endosonography Division, Pancreas Translational and Clinical Research Center, San Raffaele Scientific Institute IRCCS, VitaSalute San Raffaele University, Milan, Italy

2 Pathology Division, Pancreas Translational and Clinical Research Center, San Raffaele

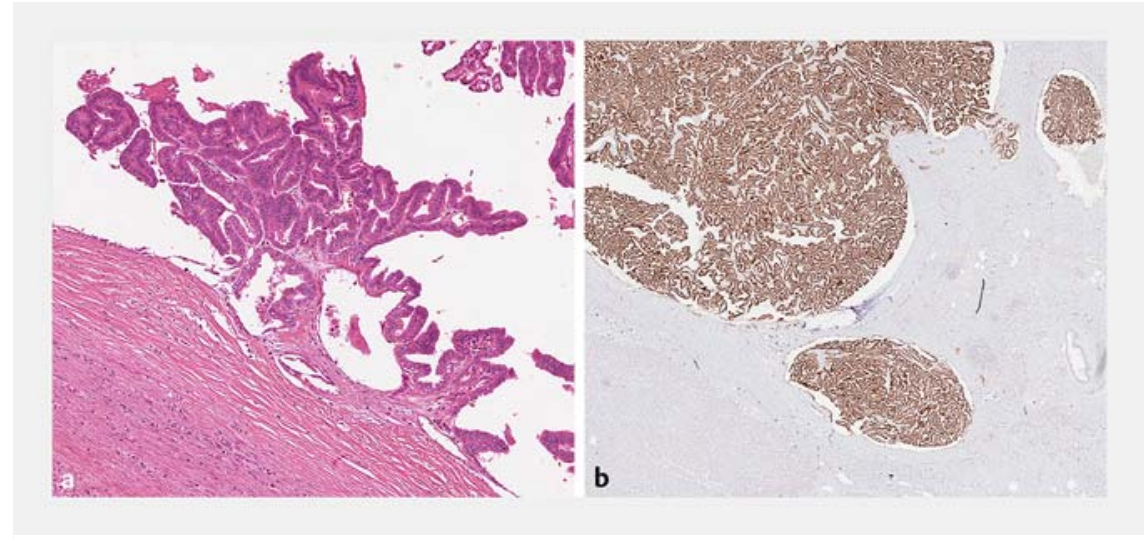

Fig. 3 Histological findings of pancreatic metastasis growing inside the main pancreatic duct. a Hematoxylin and eosin stain. $\mathbf{b}$ Immunohistochemical stain (TTF1 labels nuclei of neoplastic cells).

Scientific Institute IRCCS, Vita-Salute San Raffaele University, Milan, Italy

3 Pancreatic Surgery Division, Pancreas Translational and Clinical Research Center, San Raffaele Scientific Institute IRCCS, VitaSalute San Raffaele University, Milan, Italy

\section{Corresponding author}

\section{Gemma Rossi, MD}

Pancreato-Biliary Endoscopy and Endosonography Division, Pancreas Translational and Clinical Research Center, San Raffaele Scientific Institute IRCCS, Vita-Salute San Raffaele University, Via Olgettina 60, 20132 Milan, Italy Fax: +39-02-26435609 rossi.gemma@hsr.it

\section{References}

[1] Lubitz CC, Sosa JA. The changing landscape of papillary thyroid cancer: epidemiology, management, and the implications for patients. Cancer 2016; 122: 3754-3759

[2] Cancer stat facts: Thyroid cancer. National Cancer Institute. Available from (Accessed: 17 July 2018): http://seer.cancer.gov/statfacts/html/thyro.html

[3] Jobran R, Baloch ZW, Aviles V et al. Tall cell papillary carcinoma of the thyroid: metastatic to the pancreas. Thyroid 2000; 10 : 185-187

[4] Waters L, Si Q, Caraway N et al. Secondary tumors of the pancreas diagnosed by endoscopic ultrasound-guided fine-needle aspiration: a 10-year experience. Diagn Cytopathol 2014; 42: 738-743

[5] Smith AL, Odronic SI, Springer BS et al. Solid tumor metastases to the pancreas diagnosed by FNA: a single-institution experience and review of the literature. Cancer Cytopathol 2015; 123: 347-355

Bibliography

Endoscopy 2020; 52: E452-E453

DOI 10.1055/a-1164-6157

ISSN 0013-726X

published online 12.5 .2020

(c) 2020. Thieme. All rights reserved.

Georg Thieme Verlag KG, Rüdigerstraße 14, 70469 Stuttgart, Germany

\section{ENDOSCOPY E-VIDEOS}

https://eref.thieme.de/e-videos

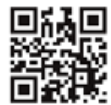

Endoscopy E-Videos is a free access online section, reporting on interesting cases and new techniques in gastroenterological endoscopy. All papers include a high quality video and all contributions are freely accessible online.

This section has its own submission website at https://mc.manuscriptcentral.com/e-videos 